\title{
PERFORMANCE OF MULTIPLE RANK MODULATION BASED ON SM-MIMO
}

\author{
Mohanad ABDULHAMID \\ AL-Hikma University, Iraq \\ moh1hamid@yahoo.com \\ Mwaniki MUCHAI \\ University of Nairobi, Kenya \\ researcher12018@yahoo.com
}

\begin{abstract}
A multiple rank modulation (MRM) scheme is proposed that provides better error performance, enhances the data rate and reduces the system demodulation complexity. Multiple input multiple output (MIMO) scheme is a technique that uses several antennas at the transmitter and receiver to minimize error and optimize data speed. MRM is a novel technique that borrows from spatial modulation-MIMO (SM-MIMO) scheme. The basic idea of this scheme involves the transmitter receiving a group of bits and subdividing them into two blocks; rank index block and signal modulation block. The rank index bit block is used to select the rank to be activated and the rank selected contains at least one active transmit antenna (TA). The signal modulation bit block is encoded in a given modulation scheme for transmission. It is then transmitted through the activated rank that contains at least one active TA. The transmitted encoded signal modulation bit block is received through the receive antenna and a receiver. The receiver estimates a rank index and the transmitted symbol from the signal received. The signal modulation bit block is finally decoded. This paper addresses the performance of MRM scheme based on error performance to run cellular fifth generation (5G). We perform and present simulation results of MIMO systems employing MRM scheme to generate bit error rate $(B E R)$ of this system.
\end{abstract}

KEYWORDS: performance, multiple rank modulation, SM-MIMO

\section{Introduction}

Today and in the coming future, the present wireless networks will have to progress in many ways to fulfill the presumptions and challenges faced. The next wireless generation communication, $5 \mathrm{G}$, is expected in providing higher data rates, typically of Gbps order, very low latency, increasing capacity and improving users quality of services (QoS) compared to current 4G Long-Term Evolution (LTE) network. With every rapid increase of smart devices and exponential rise in wireless data demand and usage, it's generating a significant burden on existing cellular network. 5G wireless systems are expected to be the solution to the current cellular networks problems. To fulfill the objectives of 5G key research, an improvements and developments have been made on several MIMO modulation techniques. These techniques have emerged as promising design of future wireless communication systems in both theoretical and practical designs (Agiwal, Roy \& Saxena, 2016, pp. 1617-1650).

One of the novel techniques proposed for MIMO systems is spatial modulation (SM) (Mesleh, Haas, Sinanovic, Wook \& Yun, 2008, pp. 2228-2241; Mietzner, 
Schober, Lampe, Gerstacker \& Hoeher, 2009, pp. 87-107; Renzo, Haas \& Grant, 2011, pp. 182-191).

In this scheme only one antenna is active among the transmitting antenna array during the transmission period hence interchannel interference (ICI) is avoid. Also the use of transmit antenna index as an information source increases overall spectral efficiency (SE) (Jeganathan, Ghrayeb \& Szczecinski, 2008, pp. 545-547; Renzo, Haas, Ghrayeb, Sugiura \& Hanzo, 2014, pp. 56-103).

Despite several advantages offered by SM scheme, there are some major concerns/limitations. Some include:

- Suboptimal spectral efficiency. Due to the fact that some TA remain inactive during transmission period, SM offers a lower output than spatial multiplexing in conventional MIMO. It therefore implies that $\mathrm{SM}$ requires a larger number of TA compared to spatial multiplexing to achieve the same SE. Another factor that can lead to low SE is the factor that SM only offers a logarithmic increase of data rate with the number of TAs compared to Vertical Bell Labs SpaceTime Architecture (V-BLAST). This limits SM to achieve high spectral efficiencies for practical numbers of TAs.
- Favorable propagation conditions. The efficiency of SM mainly depends on the channel impulse of the transmitter to receiver links being sufficiently different from each other. If this is not the case, the SM standard might not yield adequate performance in terms of error performance and energy efficiency (EE).

Based on the conventional SM, several novel schemes have being proposed. One such novel scheme is multiple rank modulation (MRM) (Akoun \& Hongjun, 2018).

Unlike in spatial modulation, where the TA is an information source, MRM utilizes the index of a multiple rank activated among a group of multiple ranks as an information source. The received signal with plurality of bit at the transmitter is altered in a modulation signal domain and a ranking domain. In the modulation signal domain, a signal modulation bit block is encoded on a signal constellation corresponding to a certain modulation scheme. In the ranking domain, a rank bit block is encoded according to the index of a rank to be activated. The selected rank may contain one or more TA but each rank contains a different number of antennas from the other. All the transmit antennas in the activated rank are used to transmit the same information. Figure no. 1 illustrates block diagram of MRM method.

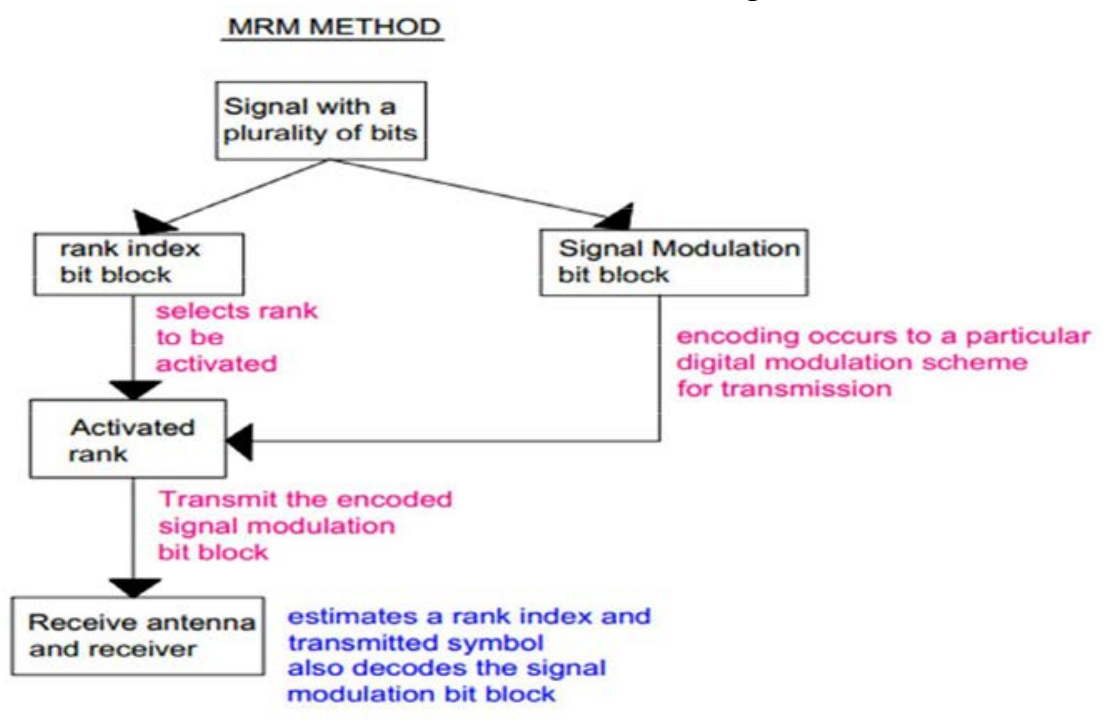

Figure no. 1: MRM method block diagram 
Unlike in SM where the uniqueness of each transmit to receive wireless channel is made use for data communication, MRM utilizes multiple channels shapes that are provided by the ranks having different numbers of transmit antennas to determine the ranking information. Therefore the ranking information will be detected even if the channels look alike as long as the number of received channels is known.

Besides the rank index being used as a source of information, MRM also uses signal modulation constellation diagram as an information source. Signal modulation and ranking modulation are applied to the input signal bits. Bits that correspond to the rank bit block are encoded by ranking modulation where a vector signal having as many symbols as the total number of antennas is generated, that is a signal modulation symbol value is set at the positions matching to the index of the rank to be activated. Bits that correspond to the signal modulation bit block in the input signal are encoded by signal modulation where 0 s are set at the symbol positions matching to the inactive antennas in the vector signal.

The number of transmitting antennas is the rank value and signal modulation bit block is mapped corresponding to antennas in the selected active rank.
Maximum likelihood (ML) algorithm is used to estimate how many antennas actually transmitted and by doing so estimates the activated rank. This reduces the complexity of the receiver compared to algorithm used for conventional MIMO systems. In MRM, the detector estimates a rank index and a transmitted symbol from the received signal. The ranking demodulator decodes a rank bit block using the estimated rank index and decodes a signal modulation bit block using the estimated transmitted symbol. The original signal is recovered by combining the rank bit block with the signal modulation bit block.

A flawless representation of MRM scheme activates at least one transmit antenna in a MIMO system to transmit the symbol. This allows a free inter-channel interference (ICI) and reduces spatial error. MRM also offers high network efficiency in terms of additional reliability while taking advantages of conventional SM.

\section{Multiple Rank Modulation Model}

Figure no. 2 represents the proposed model of a MIMO system using MRM, where $\mathrm{N}_{\mathrm{t}}$ and $\mathrm{N}_{\mathrm{r}}$ represent number of transmit and receive antennas respectively.

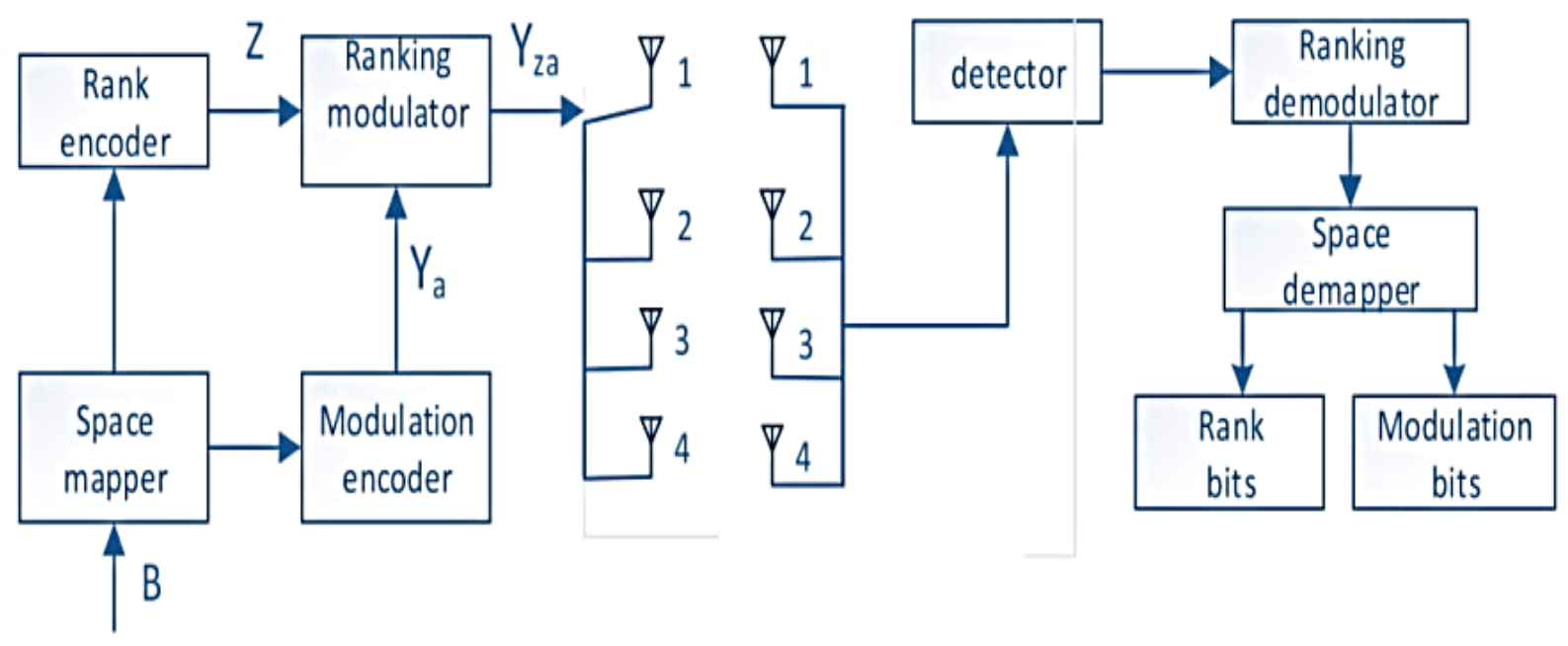

Figure no. 2: MRM system model 
The transmitter, comprising of space mapper, signal modulation encoder, rank index encoder, ranking modulator and group of transmit antennas, receives an input signal with a plurality of bits from the binary source. The space mapper separates the input signal into a rank bit block and a signal modulation bit block. Signal modulation encoder encodes the signal modulation bit block on a signal modulation scheme according to a particular digital modulation scheme, e.g. PSK (BPSK or QPSK), QAM.

The rank bit block selects the rank to be activated and the rank index encoder encodes the rank bit block as per the index of activated rank. The rank activated should contain at least one or more TAs.

The ranking modulator receives the encoded signal modulation bit block from signal modulation encoder and the encoded rank bit block from the rank index encoder. It spatially modulates the input signal to a signal with $\mathrm{N}_{\mathrm{t}}$ symbols. The ranking modulator works by applying a signal modulation constellation value to a symbol position matching $1 \mathrm{~s}$ of the modulated input signal to the active antenna in the activated rank and 0s of the modulated input signal to remaining inactive antennas. The group of transmit antennas send the modulated signal to the receiver through the activated rank at any particular time interval as per the active antennas in that particular rank.

The receiver comprises of receiver antennas, detector, ranking demodulator and space demapper. The receiver antennas receive signals from transmit antennas. This signal is then passed to the detector. The detector uses maximum likelihood (ML) algorithm to estimate the index of the active rank and the transmitted symbol. The ML algorithm estimates how many antennas actually transmitted and by doing so estimates the active rank since the number of antennas transmitting is the rank index of the activated rank (rank value). This reduces the complexity of the receiver compared to conventional MIMO systems. MRM can also use the power of received signal to decode the rank index.

The ranking demodulator decodes the rank bit block using the estimated rank index and decodes the signal modulation bit block using the estimated transmitted symbol. The space demapper demaps the rank bits according to the transmit rank and the modulation bits are demapped according to the modulation symbol vector. The original signal is obtained by combining the rank bit and modulation bits.

\section{Simulation Results}

A simulation of MRM system is implemented using MATLAB. A system with 2 transmit antennas, 4 receive antennas and at a data rate of $3 \mathrm{bits} / \mathrm{s} / \mathrm{Hz}$ MRM model is simulated. The MRM code is to implement a MRM model. The bit error rate (BER) results of the proposed systems are obtained under different number of symbol per frame (N) and different signal to noise ratio $\left(\mathrm{SNR}=\mathrm{E}_{\mathrm{b}} / \mathrm{N}_{\mathrm{o}}\right.$ ). The modulation scheme used is BPSK modulation.

The obtained BER vs SNR results are compared with different transmission schemes that are SM, V-BLAST and Adaptive Generalized Space Shift Keying (GSSK) to show the error performances of MRM scheme under the same spectral efficiency of 3bits/s/Hz.

The results obtained are used to plot BER vs $E_{b} / N_{0}$ curves. This is done for $N=10^{\wedge} 4$ and for $N=10^{\wedge} 6$. For each case the graphs are plotted as shown in Figure no. 3 and Figure no. 4. 


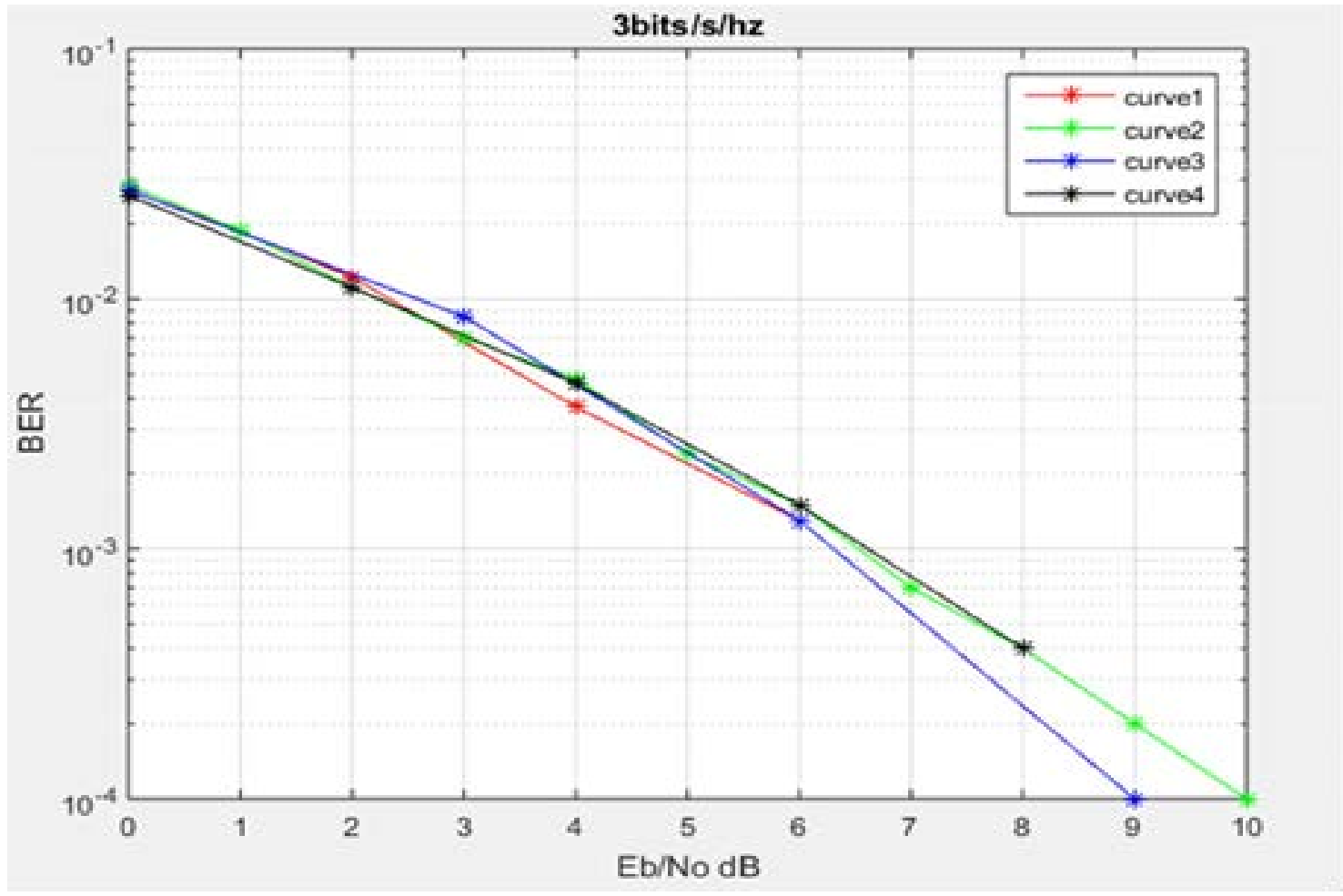

Figure no. 3: BER versus $S N R$ for $N=10^{M}$

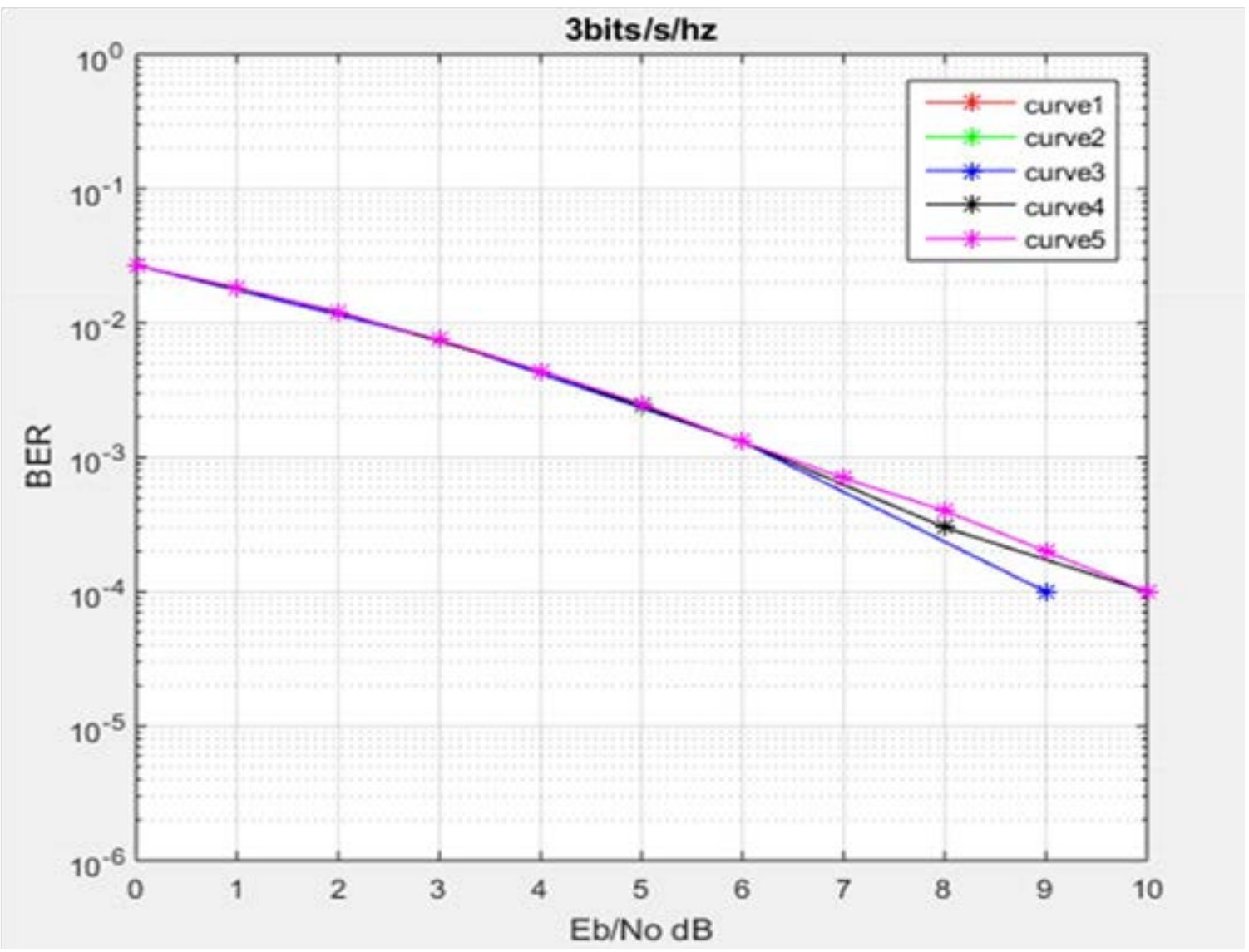

Figure no. 4: $B E R$ versus $S N R$ for $N=10^{\wedge} 6$ 
Figure no. 5 illustrates the MRM performance versus SM, GSSK and V-BLAST for the same rate of information transmitted across the channel. The data rate is set at $3 \mathrm{bits} / \mathrm{s} / \mathrm{Hz}$.

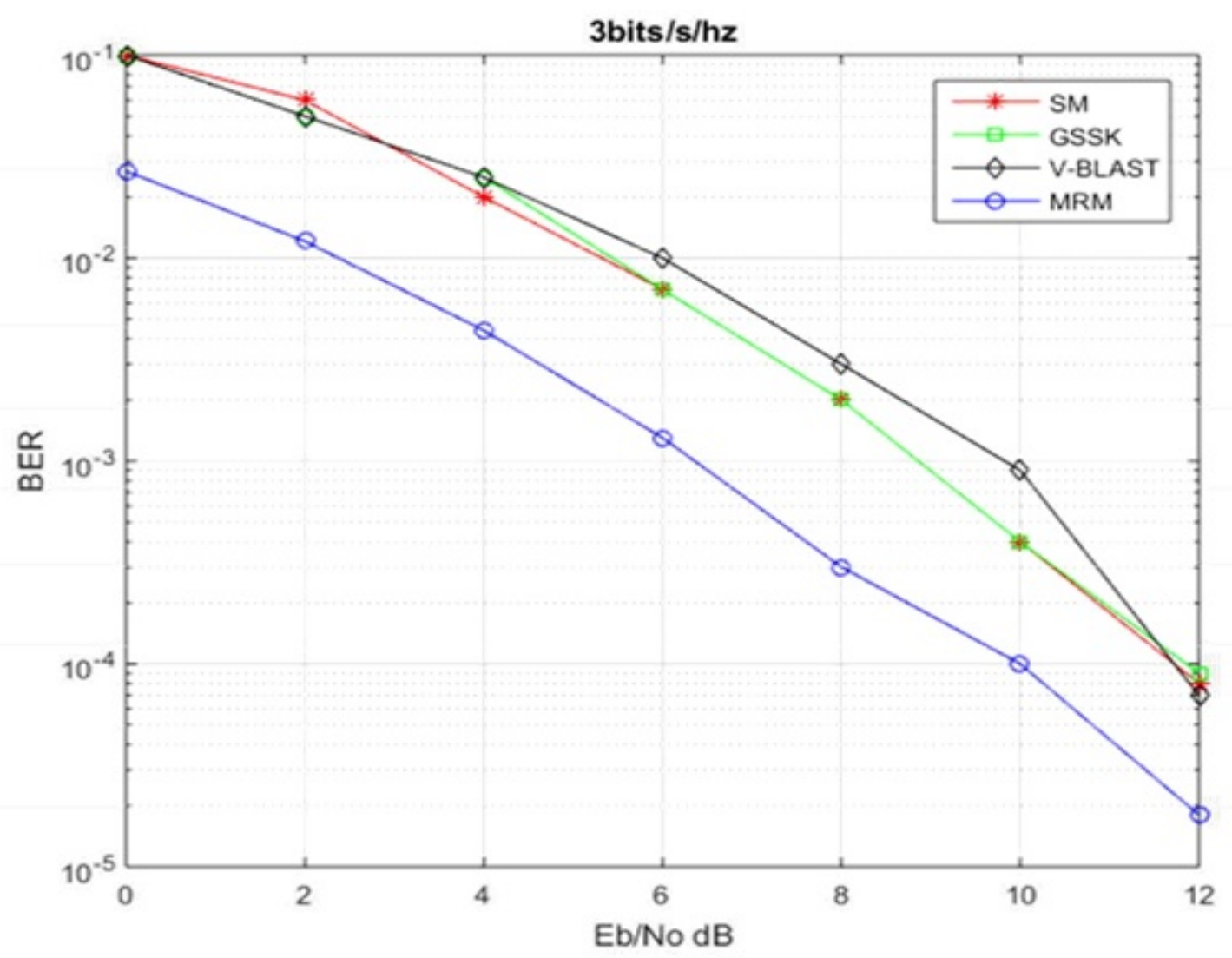

Figure no. 5: BER versus SNR for SM, GSSK, V-BLAST and MRM

V-BLAST shows poor performance as compared to SM and GSSK at same spectral efficiency. The performance of MRM shows a major improvement over SM and GSSK scheme. For a BER of $10^{-3}$ and at a 3bits $/ \mathrm{s} / \mathrm{Hz}$ transmission, MRM outperforms SM and GSSK scheme by around 2dB and also outperforms V-BLAST by around 3db. Also for $10^{-4}$, at same spectral efficiency, MRM outperforms SM, GSSK and V-BLAST by around $1.6 \mathrm{~dB}$.

\section{Conclusion and Discussion}

The concept of multiple rank modulation is proposed to relieve detection problem experienced in conventional SM. It essentially depends on favorable propagation conditions to distinguish signals at the receiver. As a result, lack of scattering in propagation environment leads to poor error performance and poor EE. The novelty of MRM is to use multiple channels shapes to determine the ranking information since each rank has a different number of channels differs from the rest of rank and therefore the ranking information will be detected even if channels look alike as long as the numbers of received channels are known. From the results obtained, MRM offers better error performance compared to conventional SM, since error in the detection of spatial bits is negligible since the channel variances among the ranking domains have a wide gap.

With the expectation of $5 \mathrm{G}$ wireless networks, MRM technique is a possible candidate for 5G wireless networks. MRM technique can offer low complexity, improved spectral and energy efficiency to be used in design of $5 G$ wireless networks. 


\section{REFERENCES}

Agiwal, M., Roy, A., \& Saxena, N. (2016). Next generation 5G wireless networks: A comprehensive review. IEEE Communication Surveys and Tutorials, Vol. 18, No. 3, 1617-1650.

Akoun, P., \& Hongjun, X. (2018). A Multiple Rank Modulation System. US Patent 2018/0269944 A1.

Jeganathan, J., Ghrayeb, A., \& Szczecinski, L. (2008). Spatial modulation: optimal detection and performance analysis. IEEE Communications Letters, Vol. 12, No. 8, 545-547.

Mesleh, R., Haas, H., Sinanovic, S., Wook, C., \& Yun, S. (2008). Spatial modulation. IEEE Transactions on Vehicular Technology, Vol. 57, No. 4, 2228-2241.

Mietzner, J., Schober, R., Lampe, L., Gerstacker, W., \& Hoeher, P. (2009). Multiple-antenna techniques for wireless communication-a comprehensive literature survey. IEEE Communication Surveys and Tutorial, Vol. 11, No. 2, 87-107.

Renzo, M., Haas, H., \& Grant, P. (2011). Spatial modulation for multiple-antenna wireless systems: A survey. IEEE Communication Magazine, Vol. 49, Issue 12, 182-191.

Renzo, M., Haas, H., Ghrayeb, A., Sugiura, S., \& Hanzo, L. (2014). Spatial modulation for generalized MIMO: challenges, opportunites and implementation. Proceedings of the IEEE, Vol. 102, No. 1, 56-103. 